\title{
THE PROGNOSTIC ROLE OF IMMUNOHISTOCHEMISTRY IN PRIMARY BONE TUMORS
}

doi: $10.2478 /$ rojost-2018-0023

\author{
Z. Pánti, ${ }^{1,2}$, R. Ene ${ }^{1,2}$, M. Nica ${ }^{1,2}$, M. Popa ${ }^{1,2}$, M. Pleniceanu ${ }^{1}$, M. Cîrstoiv ${ }^{2,3}$, C. Cîrstoiu ${ }^{1,2}$ \\ ${ }^{1}$ Orthopedics and Traumatology Department, University Emergency Hospital, Bucharest, Romania \\ 2 "Carol Davila" University of Medicine and Pharmacy, Bucharest, Romania \\ ${ }^{3}$ Obstetrics and Gynecology Department, University Emergency Hospital, Bucharest, Romania
}

In the last decades, immunohistochemistry $(\mathrm{IHC})$ has shown an important role in tumor differential diagnosis and has proven its prognostic role.

Due to the various histological subtypes of primary malignant bone tumors, its role will be more important in the future. Based on recent studies, tumor growth, cell mobility, and metastases strongly correlate to the survival of these patients. An increased number of specific $\mathrm{IHC}$ markers have been used to determine the aggressiveness of the metastatic capacity of these tumors in enhancing early specific oncological therapies.

This study presents 15 cases of primary malignant bone tumors, over a period of 4 years (2014-2018), from the Orthopedics and Traumatology Department of the University Emergency Hospital in Bucharest, where we evaluated the proliferation index using $\mathrm{Ki} 67 \mathrm{IHC}$ marker, tissue remodelation, the presence of necrosis in osteosarcoma cases in which chemotherapy was applied, and the aspect of the cytoskeleton with vimentin. Besides the histological aspect, we evaluated the tumor site, invasion and extension to the surrounding tissues (Computer Tomography, Magnetic Resonance) and vascularization with angiography, all these properties having an important prognostic role.

Our findings were similar to other research papers in literature; showing that a fast growing and high proliferation index with increased cell mobility has a worse prognosis. Pulmonary metastases occurred in a relative short time in high-grade osteosarcomas, despite the chemotherapy, and multiple metastases were present at 6 months in a dedifferentiated chondrosarcoma case, in which the high-grade sarcoma was an osteosarcoma.

Based on the literature, we also think that some specific markers might have multiple roles regarding the tumor growth local invasion and metastasis.

Keywords: immunohistochemistry, bona sarcomas, prognostic 